ESAIM: PROCEEDINGS, October 2007, Vol. 22, 127-131

Gabriel Caloz \& Monique Dauge, Editors

\title{
3D DYNAMIC MICROMAGNETIC SIMULATIONS OF SUSCEPTIBILITY SPECTRA IN SOFT FERROMAGNETIC PARTICLES
}

\author{
Fabrice Boust $^{1}$, Nicolas Vukadinovic $^{2}$ And Stphane Labbé ${ }^{3}$
}

\begin{abstract}
The high-frequency properties of small 3D ferromagnetic elements are investigated by means of micromagnetic simulations. The dynamic susceptibilty spectra associated with two types of submicrometer-size elements: a cylindrical dot with a vortex state and an eye shape particle with a bidomain state are reported. In these confined structures, the spectra reveal a rich variety of modes which are identified.

Résumé. Les propriétés hautes fréquences de petits éléments ferromagnétiques 3D ont été étudiées l'aide de simulations micromagnétiques. Les spectres de susceptibilité dynamique associés à deux types d'éléments de taille submicrométrique : un plot cylindrique présentant un état vortex et une particule en forme d'oeil présentant un état bidomaine sont reportés. Dans ces structures confinées, les spectres révèlent une riche variété de modes qui sont identifiés.
\end{abstract}

\section{INTRODUCTION}

The magnetic properties of micro-and submicrometer-sized objects, are today under great consideration mainly due to their potential applications in high-density storage media, magnetic field sensors, spin electronic devices, and microwave technologies [1]. For these applications, a continuing request for miniaturization and an increase of the operating frequency are the two main challenges. Generally, these 3D magnetic objects present a non uniform magnetic configuration and the analysis of their dynamic behavior is not a trivial task.

The purpose of the present paper is to demonstrate the relevance of the full 3D dynamic micromagnetic simulations for probing the high-frequency linear response of small magnetic elements. Two illustrative examples are considered, namely, a cylindrical dot supporting a vortex state and an eye shape particle possessing a bidomain state.

\section{BRIEF RECALL ON MAGNETIZATION DYNAMICS}

The term of magnetization dynamics covers two different regimes corresponding, respectively, to small and large amplitude motions. Large amplitude motions occur during the magnetization reversal process induced by an external magnetic field. On the other hand, small amplitude motions refer to magnetization oscillations around the equilibrium state. In this paper, this last aspect is considered. In the regime of small amplitude motions, the high-frequency response of magnetic systems $\delta \mathbf{m}$ to a weak magnetic field $\delta \mathbf{h}$ is characterized by

\footnotetext{
1 Onera, 29 Avenue de la Division Leclerc, 92320 Châtillon, France

2 Dassault Aviation, 78 quai Marcel Dassault, 92552 St-Cloud, France

3 Laboratoire de Mathématiques, Bât. 425, Université Paris 11, 91405 Orsay Cedex, France
}

(C) EDP Sciences, SMAI 2007 
the linear dynamic susceptibility tensor $\bar{\chi}$, by

$$
\bar{\chi} \delta \mathbf{h}=\frac{1}{|\Omega|} \int_{\Omega} \delta \mathbf{m},
$$

where $\Omega$ is the domain occupied by the sample. The frequency dependence of the full susceptibility tensor corresponds to the dynamic susceptibility spectra. In recent years, two approaches have been considered for computing the dynamic susceptibility spectra of nonuniform magnetization distributions. Theses approaches are distinguished by solving the Landau-Lifshitz (LL) equation for magnetization motion either in the time domain or in the frequency domain. The former method is based on the direct integration of the LL equation in the presence of a weak uniform temporal excitating field. The time-frequency transform of the response and the excitation gives access to the dynamic susceptibility. This technique was used to compute the high-frequency dynamics of thin films (2D systems) [2] and nano-sized objects (3D systems) [3]. The latter method consists in linearizing the LL equation around the equilibrium magnetization configuration and assuming harmonic time dependences for the weak uniform exciting field $\delta \mathbf{h}$ and for the dynamic magnetization response $\delta \mathbf{m}$. The dynamic susceptibility response is then deduced by solving a complex dense linear system in the frequency domain. This technique was used to compute the high-frequency response of thin films with nonuniform magnetization [4] and 3D particles of different shapes and sizes [5,6]. This frequency-domain method is considered in this paper. The results of micromagnetic simulations presented hereafter were computed using the 3D EMicroM and SMicroM codes (see Section). The used material parameters are typical for isotropic Permalloy, namely, $M_{s}=8.10^{5} \mathrm{~A} / \mathrm{m}, A=1.310^{-11} \mathrm{~J} / \mathrm{m}$, the gyromagnetic ratio $\gamma=1.7610^{11} \mathrm{~s}^{-1} \mathrm{~T}^{-1}$, and the damping parameter $\alpha=0.025$.

\section{Cylindrical particles with a vortex state}

It has been clearly stated that the equilibrium magnetic configuration within a soft ferromagnetic cylindrical dot depends on the nature of the magnetic material (mainly through $M_{s}$ and $A$ ) and on geometrical properties, the dot radius $R$ and the dot thickness $L_{z}$. Among the rich variety of nonuniform micromagnetic states, the vortex-like structure appears in dots satisfying $L_{z}>\Lambda$ and $R>>\Lambda$ where $\Lambda=\sqrt{2 A /\left(\mu_{0} M_{s}^{2}\right)}$ is the exchange length. Such a vortex structure can be viewed as a curling in-plane magnetic configuration with a central region, the vortex core, in which the magnetization is tilted out of the dot plane (Fig. 1(a)).
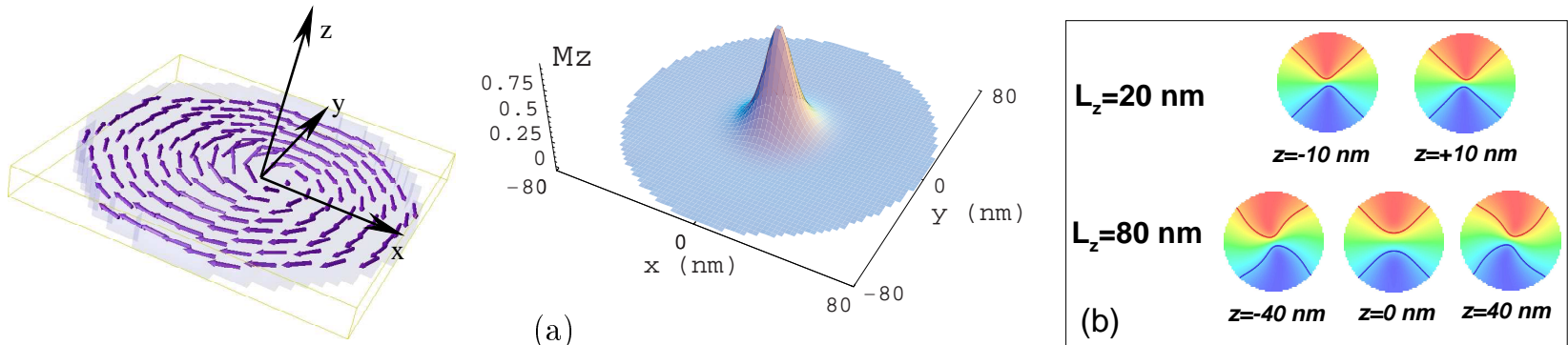

FIGURE 1. 3D static micromagnetic simulations for nanodots with radius $R=80 \mathrm{~nm}$ supporting a vortex magnetic structure at the remanent state. (a) Representation of the vortex-like structure and coordinate system. (b) $M_{x}$ maps for two thicknesses $L_{z}=20 \mathrm{~nm}$ and $L_{z}=80 \mathrm{~nm}$. The color maps correspond to views in the $(\mathrm{x}, \mathrm{y})$ plane at different $\mathrm{z}$ positions (high level in red and low level in blue).

In order to analyze the role played by the dot thickness on the linear magnetic excitations, 3D micromagnetic simulations were performed using a dot radius $R=80 \mathrm{~nm}$ and two dot thicknesses $L_{z}=20 \mathrm{~nm}$ and $L_{z}=80 \mathrm{~nm}$, respectively [7]. Figure 1(b) represents the x-component of the static magnetization $M_{x}$ in the $(x, y)$ plane for 
nanodots with $L_{z}=20 \mathrm{~nm}$ and $L_{z}=80 \mathrm{~nm}$ and at different $\mathrm{z}$ positions. The spiral spin arrangement is observed in which $M_{x}$ varies continuously from negative values (blue color) to positive ones (red color) as we turn around the dot center. For the thinnest nanodot, the maps of $M_{x}$ are nearly identical between the lower and upper dot surfaces. As the dot thickness increases, a twist appears between the two opposite surfaces in order to ensure a best flux closure between the end surface magnetic charges. It is worth noting that the vortex core radius depends significantly of the z-coordinate with the smallest values at the dot surfaces. Furthermore, a sizeable radial component of the magnetization exists at the dot surfaces for the thickest nanodots.

Figure 2 shows the imaginary part of the computed dynamic susceptibility spectra, in-plane element $\chi_{x x}^{\prime \prime}$. For the nanodot with thickness $L_{z}=20 \mathrm{~nm}$ (Figure 2(a)), three magnetic excitations, labeled (1), (2) and (3) respectively, are observed. The spatial distribution of resonance modes (color insets) indicates that the lowfrequency resonance peak (resonance frequency $f_{r}=0.94 \mathrm{GHz}$ ) arises from the spins localized within the vortex core. The resonance line $(2)\left(f_{r}=10.2 \mathrm{GHz}\right)$ results from spin regions with high values of $M_{y}$ or $M_{z}$ (excluding the peripheral zone of nanodots) whereas the resonance line $(3)\left(f_{r}=12.2 \mathrm{GHz}\right)$ is due to the excitation of spin areas with high values of $M_{y}$ outside the vortex core. For this dot thickness, the vortex-core resonance frequency can be predicted by an analytical model assuming the invariance of the vortex magnetic configuration along the dot normal (limit of flat nanodots, $L_{z} \leqslant \Lambda$ ) [8]. It should be underlined that the vortex-core resonance was detected experimentally by means of ferromagnetic resonance measurements and the resonance frequency was found in very good agreement with the computed one [3].

For a thicker nanodot $\left(L_{z}=80 \mathrm{~nm}\right)$, a new magnetic excitation, labeled $\left(1^{\prime}\right)$, appears $\left(f_{r}=3.6 \mathrm{GHz}\right)$. The spatial distribution of the resonance modes shows that the resonance lines $(1)$ and $\left(1^{\prime}\right)$ correspond to vortex core spin excitations. For both modes, a twisted dynamic structure develops between the upper and lower dot surfaces. These modes appear nonuniform along the z-axis, the resonance mode $\left(1^{\prime}\right)$ being strongly confined near the dot surfaces. The resonance line $\left(1^{\prime}\right)$ depends strongly on dot thickness and cannot be predicted using the above cited analytical model. The resonance frequencies of modes (2) and (3) vary weakly with dot thickness.
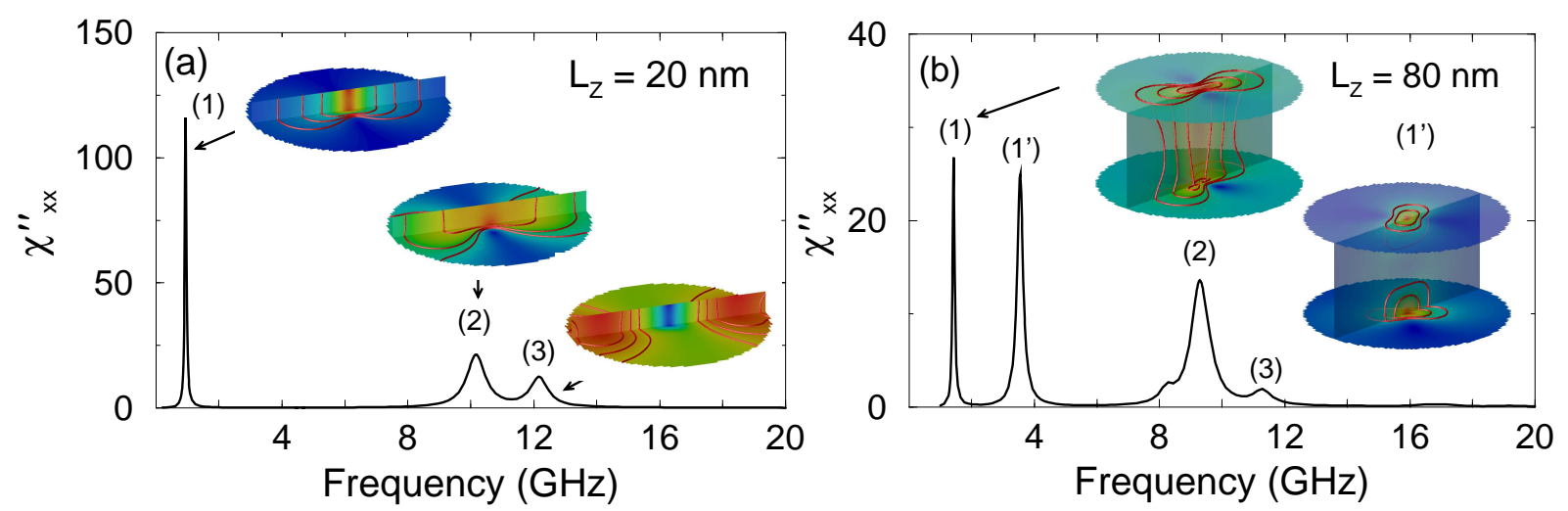

FigURE 2. 3D dynamic micromagnetic simulations of the dynamic susceptibility (imaginary part of the in-plane element $\chi_{x x}$ ) for nanodots with radius $R=80 \mathrm{~nm}$ and for two dot thicknesses $L_{z}=20 \mathrm{~nm}$ and $L_{z}=80 \mathrm{~nm}$. The spatial distribution of resonant modes within the nanodots is displayed in the color insets (high level in red and low level in blue).

\section{Eye ShAPE PARTICles With A SINGLE DOMAIN WALL}

The second example concerns an elongated particle, denoted eye shape particle. The lateral sizes are $320 \mathrm{~nm}$ $\times 640 \mathrm{~nm}$ along the $\mathrm{x}$ and $\mathrm{y}$ axes, respectively. The particle thickness is $80 \mathrm{~nm}$. In such a particle, a bidomain state including a single domain wall along the elongated direction can be stabilized (Figure 3(a)). Due to the finite size of the particle, the domain wall exhibits spatial deformations and an evolutive internal structure along the y axis. Figure 3(b) displays the dynamic susceptibility spectra along the three main axes. An high-intensity 
peak (resonance line (1), $f_{r}=1.6 \mathrm{GHz}$ ) is observed for the $\chi_{y y}$ element associated to a complicated domain wall mode. A broad resonance peak (resonance line $(2), f_{r}=9.7 \mathrm{GHz}$ ) appears in the $\chi_{x x}$ spectrum. This line arises from the excitation of an hybrid mode including domain and domain wall area. The resonance line (3) occuring at higher frequency in the $\chi_{z z}$ spectrum results from the excitation of mode spreading on the whole particle except the domain wall. A common characteristic is the existence of strongly nonuniform modes within the eye shape particle. This dynamic susceptibility spectra cannot be reproduced by analytical models.

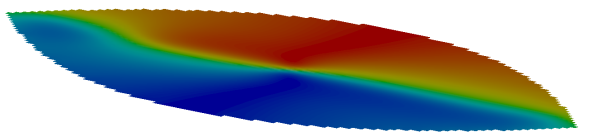

(a)

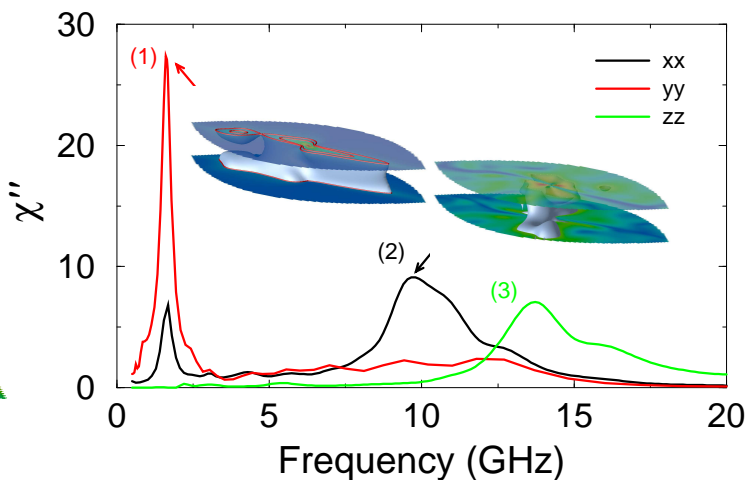

(b)

FiguRE 3. 3D micromagnetic simulations for an eye shape particle of sizes $320 \mathrm{~nm} \times 640 \mathrm{~nm}$ $\times 80 \mathrm{~nm}$ along the $\mathrm{x}, \mathrm{y}$ and $\mathrm{z}$ axes, respectively. (a) Static magnetization component $M_{y}$ at the bottom surface (positive values in red and negative values in blue). (b) Dynamic susceptibility spectra (imaginary part of the diagonal elements) with the spatial distribution of resonant modes in the color insets (high level in red and low level in blue).

\section{Concluding Remarks}

The high-frequency response of micro-and submicrometer-sized ferromagnetic elements is generally quite complicated. The geometrical confinement induces nonuniform static and dynamic magnetization distributions. In this context, full 3D micromagnetic simulations appear as a powerful tool for computing accurately the dynamic susceptibility spectra and for identifying the structure of the resonant modes. In the future, additional terms could be implemented in the dynamic micromagnetic code in order to take into account the thermal fluctuations and the spin-torque effect.

\section{REFERENCES}

[1] R. P. Cowburn. Magnetic nanodots for device applications. Journal of Magnetism and Magnetic Materials, 242-247:511-516, 2002.

[2] J.-C. Toussaint, A. Marty, N. Vukadinovic, J. Ben Youssef, and M. Labrune. A new technique for ferromagnetic resonance calculations. Computational Materials Science, 24:175-180, 2002.

[3] V. Novosad, P. Y. Fradin, P. E. Roy, K. S. Buchanan, K. Yu. Guslienko, and S. Bader. Magnetic vortex resonance in patterned ferromagnetic dots. Physical Review B, 72 024455:1-5, 2005.

[4] N. Vukadinovic, O. Vacus, M. Labrune, O. Acher, and D. Pain. Magnetic excitations in a weak-stripe-domain structure: A 2D dynamic micromagnetic approach. Physical Review Letters, 85:2817-2821, 2000.

[5] S. Labbé and P. -Y. Bertin. Microwave polarisability of ferrite particles wit nonuniform magnetization. Journal of Magnetism and Magnetic Materials, 206:93-105, 1999.

[6] C. Vaast-Paci and L. Leylekian. Numerical simulations of isolated particles susceptibilities: effects of shape and size. Journal of Magnetism and Magnetic Materials, 237:342-361, 2001.

[7] F. Boust and N. Vukadinovic. Micromagnetic simulations of vortex-state excitations in soft magnetic nanostructures. Physical Review B, 70 172408:1-4, 2004. 
[8] K. Yu. Guslienko, B. A. Ivanov, V. Novosad, Y. Otani, H. Shinia, and K. Fukamichi. Eigenfrequencies of vortex state excitations in magnetic submicron-size disks. Journal of Applied Physics, 91:8037-8039, 2002. 